CONFORMAL GEOMETRY AND DYNAMICS

An Electronic Journal of the American Mathematical Society

Volume 12, Pages 153-173 (October 14, 2008)

S 1088-4173(08)00183-5

\title{
RETURN TIMES OF POLYNOMIALS AS META-FIBONACCI NUMBERS
}

\author{
NATHANIEL D. EMERSON
}

\begin{abstract}
We consider generalized closest return times of a complex polynomial of degree at least two. Most previous studies on this subject have focused on the properties of polynomials with particular return times, especially the Fibonacci numbers. We study the general form of these closest return times. The main result of this paper is that these closest return times are metaFibonacci numbers. In particular, this result applies to the return times of a principal nest of a polynomial. Furthermore, we show that an analogous result holds in a tree with dynamics that is associated with a polynomial.
\end{abstract}

\section{INTRODUCTION}

Consider the dynamics of a polynomial $f: \mathbb{C} \rightarrow \mathbb{C}$ (see $[\mathrm{CG}$ for example). The most intuitive sequence of closest return times of a point $z_{0}$ under iteration by $f$ is defined as follows: Let $n_{1}=1$ and define $n_{k+1}$ as the least integer such that $\left|f^{n_{k+1}}\left(z_{0}\right)-z_{0}\right|<\left|f^{n_{k}}\left(z_{0}\right)-z_{0}\right|$. M. Lyubich and J. Milnor proved that there exists a real quadratic polynomial such that the closest return times of its critical point are the Fibonacci numbers $[\mathrm{LM}]$. However, these closest return times are not generally invariant if we conjugate $f$ by an affine map. Thus, it is natural to consider more general types of return times.

The return times associated with a principal nest of a polynomial are affine invariants. B. Branner and J. Hubbard studied the dynamics of polynomials with a disconnected Julia set and exactly one critical point with bounded orbit. They showed that the Fibonacci numbers occur as the return times of a principal nest of certain of these polynomials [BH, Ex. 12.4].

The above-mentioned studies are typical of previous work on return times in complex dynamics. They considered a specific sequence of return times, the Fibonacci numbers, and derived properties of polynomials with the specified return times. In contrast, the main theorem of this paper is a general result about the form of return times of a complex polynomial. There are no previously published results about the general form of closest return times of complex polynomials.

We study polynomial dynamics by associating a polynomial of degree at least 2 with a tree with dynamics (Definition 2.4 and E2]). We introduce a return nest (Definition 4.6) of a polynomial, which is a generalization of a principal nest (Definition 4.19 and $[\mathrm{L}$ ). Return nests have a combinatorial analogue in a tree

Received by the editors December 10, 2007.

2000 Mathematics Subject Classification. Primary 37F10, 37F50; Secondary 11B39.

Key words and phrases. Julia set, meta-Fibonacci, polynomial, principal nest, puzzle, return time, tree with dynamics.

(C)2008 American Mathematical Society Reverts to public domain 28 years from publication 
with dynamics. Our main theorem is that the closest return times of any return nest are meta-Fibonacci numbers (Theorem 1.2). By meta-Fibonacci numbers we mean a sequence given by a Fibonacci-type recursion, where the recursion varies with the index (see CCT for an overview).

Meta-Fibonacci numbers have not previously been considered in the context of complex dynamics. We introduce them by recalling some generalizations of Fibonacci numbers. The Fibonacci numbers are recursively defined by adding the previous two terms of the sequence: $u_{k}=u_{k-1}+u_{k-2}$. Adding the previous three terms yields the Tribonacci numbers. If we add the previous $r$ terms, we obtain $r$-generalized Fibonacci numbers ("r-bonacci numbers") Mi. The meta-Fibonacci numbers that we consider in this paper are defined by the following recursion. Let $r$ be a function of $k$, and add the previous $r(k)$ terms:

$$
n_{k}=\sum_{j=1}^{r(k)} n_{k-j} \text {. }
$$

Given $r: \mathbb{Z}^{+} \rightarrow \mathbb{Z}^{+}$with $r(k) \leq k$ for all $k$, we choose an initial condition $n_{0}$, and recursively define $n_{k}$ by Equation (1.1) for $k \geq 1$. We call the resulting sequence $\left(n_{k}\right)_{k=0}^{\infty}$ a variable-r meta-Fibonacci sequence generated by $r(k)$ [E3, Def. 1.1]. We present examples in Section 3 . In order to describe the return times of polynomials, we need to allow for the possibility that $r(k)$ is arbitrarily large. Hence, we define $r(k)$ and $n_{k}$ for all integers.

Definition 1.1 ([E3, Def. 5.1]). Let $r: \mathbb{Z} \rightarrow \mathbb{Z}^{+}$and let $\left(n_{k}\right)_{k \in \mathbb{Z}}$ be a double sequence of real numbers. We say $\left(n_{k}\right)$ is an extended variable- $r$ meta-Fibonacci sequence generated by $r(k)$ if Equation (1.1) holds for all $k \in \mathbb{Z}$. For brevity, we write "r(k)-bonacci numbers."

We describe the type of return times that we will consider. Let $f$ be a polynomial of degree at least 2. We can form a puzzle of $f$ (see \$4), which decomposes the complex plane into topological disks called puzzle pieces of $f$. We consider dynamically defined subsequences of a sequence of nested puzzle pieces. Let $\left(P_{l}\right)_{l \in \mathbb{Z}}$ be a sequence of nested puzzle pieces of $f$. A return nest is a sub-nest $\left(P_{l(k)}\right)_{k \in \mathbb{Z}}$ such that

$$
f^{n_{k}}\left(P_{l(k)}\right)=P_{l(k-1)} \quad \text { for all } k \in \mathbb{Z},
$$

where $n_{k}=\min \left\{n \geq 1: f^{n}\left(P_{l(k)}\right)=P_{m}\right.$ for some $\left.m\right\}$. We call $\left(n_{k}\right)_{k \in \mathbb{Z}}$ the return times of the return nest. In some cases, we need to modify this definition for one value of $k$ (Definition 4.6). A principal nest [L, $\S 3.1]$ is a special type of return nest. The following is our main theorem.

Theorem 1.2. The sequence of return times of any return nest of a polynomial is an extended variable-r meta-Fibonacci sequence.

Let us fix some notation. Let $f$ be a complex polynomial of degree at least 2 . We say a point is persistent if it has bounded orbit under $f$ and escapes otherwise. The filled Julia set of $f$ is the set of all persistent points of $f$, and we denote it by $\mathcal{K}(f)$. The Julia set of $f$ is the topological boundary of $\mathcal{K}(f)$, and we denote it by $\mathcal{J}(f)$. By a classical result of Fatou and Julia, the Julia set of $f$ is connected if and only if all critical points of $f$ are persistent.

Our main tool for studying polynomial dynamics is the combinatorial system of a tree with dynamics (Definition 2.4). A puzzle decomposes the plane. A tree 
with dynamics codes this decomposition and keeps track of the key features of the dynamics in a simpler setting. A polynomial with a disconnected Julia set has a canonical Branner-Hubbard puzzle $[\mathrm{BH}]$. We use this decomposition to define a canonical tree with dynamics (Definition 4.10 and [E2]). When $f$ is a polynomial with a connected Julia set, we decompose the plane into a Yoccoz puzzle $[\mathrm{H}]$. We introduce the tree with dynamics associated with a Yoccoz puzzle (Definition 4.13). Thus, trees with dynamics are a single combinatorial system for studying the dynamics of polynomials with either connected or disconnected Julia sets. Theorem 1.2 follows from an analogous result for trees with dynamics (Theorem 2.11).

Puzzles have been used in complex dynamics for some time. Branner and Hubbard defined a canonical dynamic decomposition of the plane for polynomials with exactly one escaping critical point [BH, Ch. 1.1]. Later, Branner clarified the structure of the Branner-Hubbard puzzle $[\mathrm{B}$. The author generalized this decomposition, and defined the tree with dynamics for any polynomial with a disconnected Julia set [E2, §3]. J. C. Yoccoz introduced a technique to decompose the plane using the dynamics of a polynomial with a connected Julia set, which is now called a "Yoccoz puzzle". For a quadratic polynomial, the construction was first published by Hubbard [ $\underline{\mathrm{H}}, \S 5]$. The general case was described by J. Kiwi [ $\underline{\mathrm{K}}, \S 12]$.

Trees with dynamics were introduced by R. Pérez-Marco $[\mathrm{P}-\mathrm{M}]$. The first substantive results using trees with dynamics to study polynomials with disconnected Julia sets were obtained by the author (E1] and [E2]). In the case of a polynomial with a disconnected Julia set, Theorem 1.2 is part of the author's thesis [E1. The connected case has not previously been published.

The remainder of this paper is organized as follows. In Section 2, we define trees with dynamics abstractly, and prove some preliminary results about them. We prove that a combinatorial version of Theorem 1.2 holds in any tree with dynamics (Theorem 2.11). We then give a construction of a combinatorial analogue of a return nest. Finally, we describe the initial conditions satisfied by the return times of any return nest. In Section 3, we study the properties of $r(k)$-bonacci sequences and present some examples. We derive estimates on the growth of $n_{k}$ in terms of $r(k)$. In Section 4, we prove our main theorem and define a tree with dynamics for a polynomial. We consider abstract puzzles with compatible dynamics, which are generalized Markov partitions. We show that there is a tree with dynamics associated with each abstract puzzle. This definition establishes a correspondence between puzzles and trees with dynamics. Theorem 1.2 follows from this correspondence. We outline the construction of a puzzle for a polynomial with either a disconnected Julia set or with a connected Julia set. We finish by noting some properties of a tree with dynamics of any polynomial.

\section{Trees With Dynamics}

In this section, we study trees with dynamics and prove a combinatorial version of our main theorem. The proof is straightforward once the necessary machinery is in place. We define a general tree with dynamics (\$2.1). A return chain is an analogue of a return nest in a tree with dynamics (Definition 2.8). The dynamics of return nests are the subject of 22.2 where we prove several important lemmas. We prove a version of Theorem 1.2 for return chains (Theorem 2.11) in $\$ 2.3$. We give necessary and sufficient conditions for a tree with dynamics to have a return chain (92.4). Finally, we consider a class of trees with dynamics that includes all 
trees with dynamics of polynomials $(\$ 2.5)$. The return times of these trees with dynamics satisfy certain restrictions (Proposition 2.15).

2.1. Preliminaries. A tree is a countable connected graph with every circuit trivial. We say two vertices of a graph are adjacent if there is an edge between them. We only consider trees with a particular type of order on their vertices.

Definition 2.1. A genealogical tree is a tree $T$ such that each vertex $v \in T$ is associated with a unique adjacent vertex $v^{p}$, the parent of v. Every vertex adjacent to $v$, except $v^{p}$, is called a child of $v$ and denoted by $v^{c}$.

In this paper, by "tree" we mean genealogical tree. We use the symbol $T$ to represent both the tree and its vertex set; the edge set is left implicit. We use sans serif symbols for trees and objects associated with trees. Our convention in drawing trees is that a parent is above its children (see Figure 11). So $v^{p}$ is above $v$ and any $v^{c}$ is below $v$. When it is necessary to distinguish between children of $v$ we use the notation $\mathrm{v}^{\mathrm{c}_{i}}$. We say $\mathrm{v}$ is an ancestor of $\mathrm{v}^{\prime}$ if there are vertices $\mathrm{v}_{0}, \ldots, \mathrm{v}_{n}$ such that $\mathrm{v}=\mathrm{v}_{0}, \mathrm{v}^{\prime}=\mathrm{v}_{n}$, and $\mathrm{v}_{i-1}=\mathrm{v}_{i}^{\mathrm{p}}$ for $i=1, \ldots, n$. We say $\mathrm{v}^{\prime \prime}$ is a descendant of $\mathrm{v}$ if $\mathrm{v}$ is an ancestor of $v^{\prime \prime}$.

Definition 2.1 also defines a genealogical graph. A genealogical graph is a tree if and only if any two vertices have a common ancestor and there is no vertex $v$ such that $\mathrm{v}^{\mathrm{p}}$ is a descendant of $\mathrm{v}$.

Let $T$ be a tree such that $T=\bigcup_{l \in \mathbb{Z}} T_{l}$, where $T_{l}=\left\{v \in T: v^{p} \in T_{l-1}\right\}$ for each $l$. We call each $\mathrm{T}_{l}$ a level of $\mathrm{T}$. We can inductively partition any tree $\mathrm{T}$ into levels, and this partition is unique up to a choice of $T_{0}$. Thus, the levels of $T$ are well defined up to indexing. Hereafter we will assume that any tree has its levels indexed in some manner.

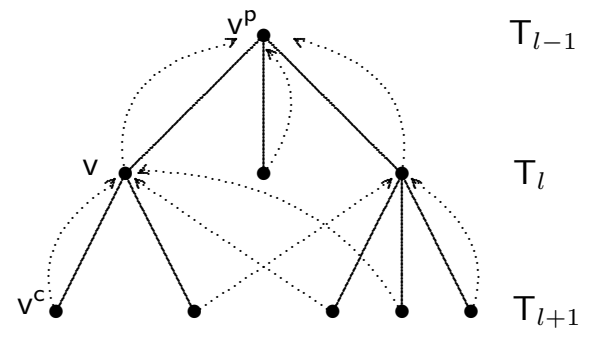

Figure 1. A tree with dynamics with $H=1$.

We consider all infinite paths in the tree that move from parent to child. Let $\mathbb{N}$ denote the non-negative integers.

Definition 2.2. Let $T$ be a tree. An end of $T$ is a sequence $\mathbf{x}=\left(x_{l}\right)_{l \in \mathbb{N}}$, where $\mathrm{x}_{l} \in \mathrm{T}_{l}$ and $\mathrm{x}_{l-1}=\mathrm{x}_{l}^{\mathrm{p}}$ for all $l$. An extended end is the analogous double sequence $\mathbf{x}=\left(\mathrm{x}_{l}\right)_{l \in \mathbb{Z}}$.

A natural metric for the extended ends of $\mathrm{T}$ is a Gromov metric:

$$
\operatorname{dist}(\mathbf{x}, \mathbf{y})=\gamma^{-L}, \quad L=\max \left\{l \in \mathbb{Z}: \mathbf{x}_{l}=\mathrm{y}_{l}\right\},
$$

for some $\gamma>1$. Any two such metrics are equivalent. We can extend such a metric to vertices of $\mathrm{T}$ by taking the minimum over all ends that contain the vertices. The set of ends of $T$ is the topological boundary of $T$ in any of these metrics. 
The dynamics that we consider are maps that preserve the genealogical structure.

Definition 2.3. Let $\mathrm{T}$ be a tree. A map $\mathrm{F}: \mathrm{T} \rightarrow \mathrm{T}$ preserves children if for all $v \in T$ the image of a child of $v$ is a child of $F(v)$. Symbolically $F\left(v^{c}\right)=F(v)^{c}$.

A children-preserving map induces a well-defined map on the set of ends of the tree. Additionally, such a map is continuous with respect to any Gromov metric. It is easy to check that if $\mathrm{F}: \mathrm{T} \rightarrow \mathrm{T}$ is a children-preserving map, then there exists $H \in \mathbb{Z}$ such that $\mathrm{F}\left(\mathrm{T}_{l}\right)=\mathrm{T}_{l-H}$ for all $l \in \mathbb{Z}$.

Definition 2.4. A tree with dynamics is a pair $(T, F)$, where $T$ is a genealogical tree and $\mathrm{F}: \mathrm{T} \rightarrow \mathrm{T}$ preserves children.

This is a very general definition. In order to be a tree with dynamics of a polynomial (Definitions 4.10 and 4.13), there are a number of additional conditions which must be satisfied (see Proposition 4.17 and [E2, Def. 4.7]).

Throughout this paper, let $(T, F)$ denote a tree with dynamics such that for some $H \in \mathbb{Z}, \mathrm{F}\left(\mathrm{T}_{l}\right)=\mathrm{T}_{l-H}$ for all $l$.

We give a few examples of trees with dynamics. Let $(T, F)$ be a tree with dynamics and let $n \geq 1$. A straightforward check of the above definitions shows that $\left(\mathrm{T}, \mathrm{F}^{n}\right)$ is also a tree with dynamics.

The following example describes the tree with dynamics of every quadratic polynomial with a disconnected Julia set (see Example 4.11).

Example 2.5 (Disconnected Quadratic Tree with Dynamics). We define a tree with dynamics as follows (see Figure 3). Define $\mathrm{T}_{-l}$ as a single vertex $\mathrm{v}_{-l}$ for $l \in \mathbb{N}$. Define $\mathrm{v}_{-l}^{\mathrm{p}}=\mathrm{v}_{-l-1}$, and $\mathrm{F}\left(\mathrm{v}_{-l}\right)=\mathrm{v}_{-l-1}$ for $l \in \mathbb{N}$. Give $\mathrm{v}_{0}$ two children: $\mathrm{v}_{0}^{\mathrm{c}_{0}}$ and $\mathrm{v}_{0}^{\mathrm{c}_{1}}$, so $\mathrm{T}_{1}=\left\{\mathrm{v}_{0}^{\mathrm{c}_{0}}, \mathrm{v}_{0}^{\mathrm{c}_{1}}\right\}$. Define $\mathrm{F}\left(\mathrm{v}_{0}^{\mathrm{c}_{i}}\right)=\mathrm{v}_{0}$. For $\mathrm{v} \in \mathrm{T}_{l}(l>0)$, give $\mathrm{v}$ two children $\mathrm{v}^{\mathrm{c}_{0}}, \mathrm{v}^{\mathrm{c}_{1}}$, and define $\mathrm{F}\left(\mathrm{v}^{\mathrm{c}_{i}}\right)=\mathrm{F}(\mathrm{v})^{\mathrm{c}_{i}}$. In this tree with dynamics, $H=1$.

The following example is not natural, but it is a useful source of counterexamples.

Example 2.6. Let $\mathrm{T}=\left\{(l, m) \in \mathbb{Z}^{2}\right\}$. Define $(l, m)^{\mathrm{p}}=(l-1,\lfloor m / 2\rfloor)$ for $m \geq 0$, and $(l, m)^{\mathrm{p}}=(l-1,0)$ for $m<0$ (where $\lfloor\cdot\rfloor$ is the greatest integer function). So $\mathrm{T}_{l}=\{(l, m): m \in \mathbb{Z}\}$. For any $H \in \mathbb{Z}$, define $\mathrm{F}_{H}(l, m)=(l-H, m)$. Check that $\mathrm{F}_{H}$ is child preserving (in fact it is an automorphism of $\mathrm{T}$ ). Thus, $\left(\mathrm{T}, \mathrm{F}_{H}\right)$ is a tree with dynamics. On the other hand, we can define an infinite-to-one map by $\mathrm{G}_{H}(l, m)=(l-H, 0)$ for any $H \in \mathbb{Z}$. Nonetheless $\mathrm{G}_{H}$ is child preserving, so $\left(\mathrm{T}, \mathrm{G}_{H}\right)$ is a tree with dynamics.

The above example is not the tree of any polynomial, since it does not satisfy the first 3 conclusions of Proposition 4.17.

2.2. Dynamics of ends. In this paper, the main type of dynamics that we consider are the returns of an end of a tree with dynamics to itself. Let $x_{L}$ be a vertex of an extended end $\mathbf{x}=\left(\mathrm{x}_{l}\right)$. We say $\mathrm{F}^{n}\left(\mathrm{x}_{L}\right)$ returns $($ to $\mathbf{x})$ if $\mathrm{F}^{n}\left(\mathrm{x}_{L}\right)=\mathrm{x}_{m}$ for some $m \in \mathbb{Z}$ and $n \geq 1$. We say $\mathrm{F}^{n}\left(\mathrm{x}_{L}\right)$ is the first return of $\mathrm{x}_{L}$ (to $\left.\mathbf{x}\right)$ if $\mathrm{F}^{n}\left(\mathrm{x}_{L}\right)$ returns and $n \geq 1$ is the minimal iterate that returns; we call $n$ the first return time of $\mathrm{x}_{L}$.

Lemma 2.7. Let $\mathbf{x}=\left(\mathrm{x}_{l}\right)_{l \in \mathbb{Z}}$ be an extended end of $(\mathrm{T}, \mathrm{F})$. Let $\mathrm{x}_{L} \in \mathbf{x}$. If $\mathrm{F}^{n}\left(\mathrm{x}_{L}\right)$ returns for some $n \geq 1$, then $\mathrm{F}^{n}\left(\mathrm{x}_{l}\right)$ also returns for every $l<L$. 
Proof. It suffices to show that $\mathrm{F}^{n}\left(\mathrm{x}_{L-1}\right)$ returns. Say that $\mathrm{F}^{n}\left(\mathrm{x}_{L}\right)=\mathrm{x}_{M}$. Since $\mathrm{F}$ preserves children, it also preserves parents. We have $\mathrm{F}^{n}\left(\mathrm{x}_{L-1}\right)=\mathrm{F}^{n}\left(\mathrm{x}_{L}^{\mathrm{p}}\right)=\mathrm{x}_{M}^{\mathrm{p}}=$ $\mathrm{x}_{M-1}$ by indexing of the end.

We introduce a simple combinatorial object, a return chain (see Figure2). Which is a subset of an end defined using first returns. It corresponds to a return nest (Definition 4.6). Essentially this allows us to consider a "one-dimensional" system, rather than the whole tree.

Definition 2.8. Let $\mathbf{x}$ be an extended end of $\mathrm{T}$. A return chain is a sub-end $\left(\mathrm{x}_{l(k)}\right)_{k \in \mathbb{Z}}$ such that

$$
\mathrm{F}^{n_{k}}\left(\mathrm{x}_{l(k)}\right)=\mathrm{x}_{l(k-1)} \quad \text { for all } k \in \mathbb{Z},
$$

where $n_{k}$ is the first return time of $\mathrm{x}_{l(k)}$. We call $\left(n_{k}\right)_{k \in \mathbb{Z}}$ the return times of the return chain. If $l(k)=\min \left\{l: \mathrm{x}_{l(k-1)}\right.$ is the first return of $\left.\mathrm{x}_{l}\right\}$ for all $k>0$, then we call the return chain minimal.

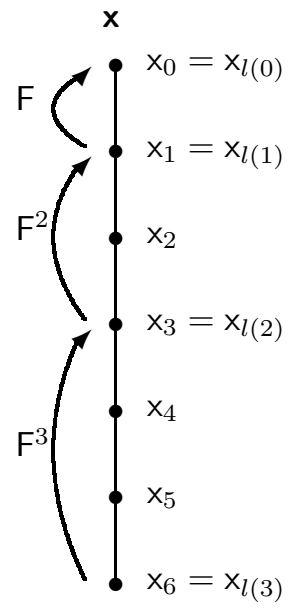

FiguRe 2. An end with a return chain marked.

Let $(\mathrm{T}, \mathrm{F})$ be a tree with dynamics such that $H \geq 1$, and let $\left(\mathrm{x}_{l(k)}\right)$ be a return chain. For each $k, \mathrm{x}_{l(k)}$ is the first return of $\mathrm{x}_{l(k+1)}$. It is possible that some $\mathrm{x}_{l(k)}$ is the first return of several $x_{l}$. In a minimal return chain, $x_{l(k+1)}$ is the first vertex of $\mathbf{x}$ below $\mathrm{x}_{l(k)}$ whose first return is $\mathrm{x}_{l(k)}$.

Lemma 2.9. Let $(\mathrm{T}, \mathrm{F})$ be a tree with dynamics such that $H \geq 1$. If $\left(n_{k}\right)_{k \in \mathbb{Z}}$ are the

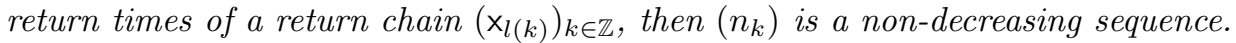

Proof. Fix $k$. We have $l(k-1)<l(k)$ since $H \geq 1$. Because $\mathrm{F}^{n_{k}}\left(\mathrm{x}_{l(k)}\right)$ is a return, $\mathrm{F}^{n_{k}}\left(\mathrm{x}_{l(k-1)}\right)$ is also a return by Lemma 2.7 Thus, $n_{k} \geq n_{k-1}$ since $n_{k-1}$ is the first return time of $\mathrm{x}_{l(k-1)}$ and first return times are minimal.

Return times are non-decreasing, but we can have $n_{k+1}=n_{k}$ for some $k$. A cascade of central returns of length $j \geq 1$ is a constant string $n_{k}=\cdots=n_{k+j-1}$ for some $k \geq 1$ (compare to $[\mathrm{L}, \S 3]$ ). The restriction on $k$ is to avoid trivial cascades of infinite length (see Corollary 4.8). Cascades of central returns of infinite 
length, equivalently eventually constant return times, occur exactly when we have a periodic end. Thus, we code trivial dynamics by trivial return times.

Lemma 2.10. Let $\mathbf{x}$ be an extended end of $(\mathrm{T}, \mathrm{F})$. If $H \geq 1$, then the following are equivalent.

(1) The minimum period of $\mathbf{x}$ is $N$.

(2) The return times $\left(n_{k}\right)$ of some return chain of $\mathbf{x}$ satisfy $n_{k}=N$ for all $k$ sufficiently large.

(3) There is a return chain of $\mathbf{x}$, and the return times $\left(n_{k}\right)$ of every return chain of $\mathbf{x}$ satisfy $n_{k}=N$ for all $k$ sufficiently large.

Proof. $(1 \Rightarrow 2)$. If $N$ is the minimum period of $\mathbf{x}$, then there is an $L$ such that for all $l \geq L, \mathrm{~F}^{n}\left(\mathrm{x}_{l}\right) \notin \mathbf{x}$ for $n=1, \ldots, N-1$, but $\mathrm{F}^{N}\left(\mathrm{x}_{l}\right) \in \mathbf{x}$. So, the first return time of $\mathrm{x}_{l}$ equals $N$. Define $l(0)=L$. For $k \leq 0$, define $n_{k}$ as the first return time of $\mathrm{x}_{l(k)}$, and $\mathrm{x}_{l(k-1)}=\mathrm{F}^{n_{k}}\left(\mathrm{x}_{l(k)}\right)$. For $k>0$, define $n_{k}=N$, and $l(k)=l(k-1)+H N$. Then $\left(\mathrm{x}_{l(k)}\right)_{k \in \mathbb{Z}}$ is a return chain with $n_{k}=N$ for all $k \geq 0$.

$(2 \Rightarrow 3)$. Let $\left(\mathrm{x}_{l(k)}\right)_{k \in \mathbb{Z}}$ be a return chain with $n_{k}=N$ for all $k \geq K$. Let $\left(\mathrm{x}_{\hat{l}(k)}\right)_{k \in \mathbb{Z}}$ be a return chain of $\mathbf{x}$ with return times $\hat{n}_{k}$. Fix $\hat{l}(k) \geq l(K)$. Then $\hat{n}_{k} \geq N$ by Lemma 2.7. Also, we can find $K^{\prime}>K$ such that $l\left(K^{\prime}\right)>\hat{l}(k)$. So, $\hat{n}_{k}=N$ by Lemma 2.7

$(3 \Rightarrow 1)$. Let $\left(x_{l(k)}\right)_{k \in \mathbb{Z}}$ be a return chain of $\mathbf{x}$. Then the return times satisfy $n_{k}=N$ for all $k \geq K$ for some $K$. For any $l \in \mathbb{Z}$, we can find $k \geq K$ such that $l(k)>l$. Since $n_{k}=N$, we have $\mathrm{F}^{N}\left(x_{l}\right) \in \mathbf{x}$ by Lemma 2.7. Therefore $\mathrm{F}^{N}(\mathbf{x})=\mathbf{x}$, and $\mathbf{x}$ is periodic with period $N$. For $l \geq l(K), F^{n}\left(\mathrm{x}_{l}\right) \notin \mathbf{x}$ for $n=1, \ldots, N-1$ by Lemma 2.7 and minimality of $n_{K}$. Therefore, $N$ is the minimum period of $\mathbf{x}$.

2.3. Main theorem for trees with dynamics. We are ready prove our main theorem for trees with dynamics, which is a combinatorial version of Theorem 1.2 .

Theorem 2.11. Let $(\mathrm{T}, \mathrm{F})$ be a tree with dynamics such that $H \geq 1$. The return times of any return chain of $(\mathrm{T}, \mathrm{F})$ are variable-r meta-Fibonacci numbers.

Proof. Let $\left(\mathrm{x}_{l(k)}\right)_{k \in \mathbb{Z}}$ be a return chain with return times $\left(n_{k}\right)_{k \in \mathbb{Z}}$. Fix $k \in \mathbb{Z}$. If $n_{k}=n_{k-1}$, then $r(k)=1$ and we are done. Otherwise, $n_{k}>n_{k-1}$ by Lemma 2.9. Thus, $n_{k}=N+n_{k-1}$ for some $N \in \mathbb{Z}^{+}$. We have

$$
\mathrm{F}^{n_{k}}\left(\mathrm{x}_{l(k-1)}\right)=\mathrm{F}^{N+n_{k-1}}\left(\mathrm{x}_{l(k-1)}\right)=\mathrm{F}^{N}\left(\mathrm{~F}^{n_{k-1}}\left(\mathrm{x}_{l(k-1)}\right)\right)=\mathrm{F}^{N}\left(\mathrm{x}_{l(k-2)}\right) .
$$

Since $\mathrm{F}^{n_{k}}\left(\mathrm{x}_{l(k)}\right)$ returns, so does $\mathrm{F}^{n_{k}}\left(\mathrm{x}_{l(k-1)}\right)$ by Lemma 2.7. Thus, $N \geq n_{k-2}$ by minimality of $n_{k-2}$. Therefore, $n_{k} \geq n_{k-1}+n_{k-2}$. If we have equality, then we are done. Otherwise, we can repeat the above argument to show that $n_{k} \geq$ $n_{k-1}+n_{k-2}+n_{k-3}$ and so on. After a finite number of repetitions of this argument we will have equality, since $n_{k}$ is finite. Therefore for some $r(k) \geq 1$,

$$
n_{k}=n_{k-1}+\cdots+n_{k-r(k)} .
$$

There is no reason that we should expect that $r(k) \leq k$. Hence, we must consider extended $r(k)$-bonacci numbers. In fact given $K, R \in \mathbb{Z}^{+}$, there is a tree with dynamics of a polynomial with a disconnected Julia set, which has a return chain with a return times $\left(n_{k}\right)$ generated by $r(k)$ such that $r(K) \geq R$ [E2, Lem. 7.12].

Our main theorem follows from the above theorem. An end of a tree with dynamics of a polynomial corresponds to a sequence of nested puzzle pieces (Definition 4.5). Thus, a return chain corresponds to a return nest (Definition 4.6). We need 
only compare Definitions 2.8 and 4.6, and verify that they define the same return times. The details of the correspondence are explained in Section 4

2.4. Constructing return chains. Say an end is recurrent if its forward iterates accumulate at itself. First, we show that every vertex of a recurrent end is the first return of some vertex of the end. Notice by definition of the metric on trees, an end $\mathbf{x}$ is recurrent if and only if for all $L \in \mathbb{Z}$ there is $K \in \mathbb{Z}$ such that $\mathrm{F}^{N}\left(\mathrm{x}_{K}\right)=\mathrm{x}_{L}$ for some $N \geq 1$.

Lemma 2.12. Let $(\mathrm{T}, \mathrm{F})$ be a tree with dynamics. Let $\mathbf{x}$ be an extended end of $\mathrm{T}$ that is recurrent under $\mathrm{F}$. For every $L \in \mathbb{Z}$, there exists $M \in \mathbb{Z}$ such that $\mathrm{x}_{L}$ is the first return of $\mathrm{x}_{M}$.

Proof. Fix $L \in \mathbb{Z}$. Since $\mathbf{x}$ is recurrent, $\mathrm{F}^{n}\left(\mathrm{x}_{K}\right)=\mathrm{x}_{L}$ for some $K \in \mathbb{Z}$ and $n \geq 1$. It is possible that $\mathrm{x}_{K}$ has returned to $\mathbf{x}$ before it returns to $\mathrm{x}_{L}$. Let $F^{N}\left(\mathrm{x}_{K}\right)=\mathrm{x}_{M}$ be the last return of $\mathrm{x}_{K}$ before it returns to $\mathrm{x}_{L}$. Then $F^{n-N}\left(\mathrm{x}_{M}\right)=\mathrm{x}_{L}$ and this is the first return of $\mathrm{x}_{M}$.

We can form a return chain of an end if and only if the end is recurrent.

Proposition 2.13. Let $(\mathrm{T}, \mathrm{F})$ be a tree with dynamics with $H \geq 1$. Let $\mathbf{x}$ be an extended end of $\mathrm{T}$ that is recurrent under $\mathrm{F}$. For any $L \in \mathbb{Z}, \mathbf{x}$ has a unique minimal return chain $\left(\mathrm{x}_{l(k)}\right)_{k \in \mathbb{Z}}$ with $l(0)=L$.

Proof. Let $l(0)=L$. For $k \leq 0$, recursively define $n_{k}$ as the first return time of $\mathrm{x}_{l(k)}$, and $l(k-1)$ by $\mathrm{x}_{l(k-1)}=\mathrm{F}^{n_{k}}\left(\mathrm{x}_{l(k)}\right)$. Notice there is no reason to believe that $l(k)$ is minimal for $k \leq 0$, nor does Definition 2.8 required it. For $k>0, \mathrm{x}_{l(k)}$ is the first return of some vertex of $\mathbf{x}$ by Lemma 2.12. There is such a vertex with least index since $H \geq 1$. We define $l(k+1)$ as the least integer such that $\mathrm{x}_{l(k)}$ is the first return of $x_{l(k+1)}$. Define $n_{k+1}$ as the first return time of $x_{l(k+1)}$. We have made no choices, so the minimal return chain we have constructed is unique.

If $\mathbf{x}$ is not recurrent, then the above construction will break down at some stage. That is, for some $K \geq 0, \mathrm{x}_{l(K)}$ will not be the first return of any vertex of $\mathbf{x}$. In this case, we define $n_{k}=r(k)=\infty$ for all $k>K$. The above construction is a combinatorial version of the construction of a principal nest $[\mathrm{L}, \S 3.1]$.

Let $\left(\mathrm{x}_{l(k)}\right)$ be a return chain of $\mathbf{x}$ with return times $\left(n_{k}\right)$ generated by $r(k)$. By Lemma 2.10, $\mathbf{x}$ is periodic if and only if $r(k)=1$ for all $k$ sufficiently large. From the above construction, $\mathbf{x}$ is non-recurrent if and only if $r(k)=\infty$ for all $k$ sufficiently large. Therefore, $r(k)$ can be thought of as an indication of the degree to which $\mathbf{x}$ is recurrent: Small $r(k)$ means that $\mathbf{x}$ is highly recurrent; large $r(k)$ means that $\mathbf{x}$ is weakly recurrent.

2.5. Rooted trees. We have been working with quite general trees with dynamics. We now restrict our attention to trees with a distinguished vertex, a root. The tree with dynamics of any polynomial has a root (Proposition 4.17(3)).

Definition 2.14. Let $\mathrm{T}$ be a tree, and let $\mathrm{v}_{0} \in \mathrm{T}$. We call $\mathrm{v}_{0}$ the root of $\mathrm{T}$, if every ancestor of $v_{0}$ has exactly one child and $v_{0}$ has more than one child. We call a tree with a root a rooted tree.

The root of a tree is unique, if it exists. In a rooted tree, we index the levels so that $T_{0}=\left\{v_{0}\right\}$, where $v_{0}$ is the root of $T$. The ancestors of the root are a countable line graph. Thus, $\mathrm{T}_{-l}$ is a single vertex $\mathrm{v}_{-l}$ for $l \in \mathbb{N}$. The boundary of a rooted tree 
has the topology of a Cantor set (compact, perfect, and totally disconnected) union one point (corresponding to $\lim v_{-l}$ ). The dynamics of a rooted tree are Lipschitz continuous. There are restrictions on return times in a rooted tree.

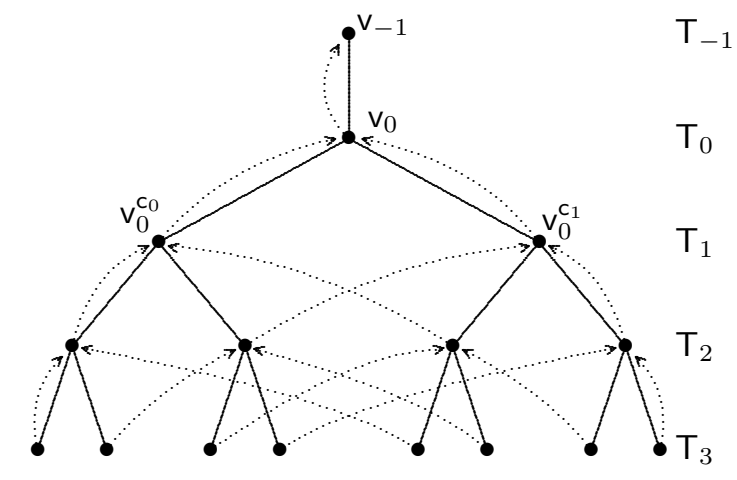

Figure 3. A rooted tree with dynamics. This is the tree with dynamics of every quadratic polynomial with a disconnected Julia set (see Example 4.11).

Proposition 2.15. Let $(\mathrm{T}, \mathrm{F})$ be a tree with dynamics such that $H \geq 1$. Let $\left(\mathrm{x}_{l(k)}\right)_{k \in \mathbb{Z}}$ be a return chain of $\mathbf{x}$ with return times $\left(n_{k}\right)$ generated by $r(k)$. If $\mathrm{T}$ is rooted, then there exists $K \in \mathbb{Z}$ such that $n_{k}=1$ and $r(k)=1$ for all $k \leq K$.

Proof. Since $\mathrm{T}$ is rooted, we have $\mathrm{T}_{-l}=\left\{\mathrm{v}_{-l}\right\}$ for all $l \in \mathbb{N}$. Thus, $\mathrm{x}_{-l}=\mathrm{v}_{-l}$, and $\mathrm{F}\left(\mathrm{x}_{-l}\right)=\mathrm{x}_{-l-H}=\mathrm{v}_{-l-H}$ for all $l \in \mathbb{N}$. We can find $K$ such that $l(K) \leq H$. Then $\mathrm{F}\left(\mathrm{x}_{l(K)}\right)=\mathrm{x}_{l(K)-H}$, so $n_{K}=1$. Since return times are non-decreasing by Lemma 2.9. $n_{k}=1$ for all $k \leq K$. By definition, $r(k)=1$ for all $k \leq K$.

This proposition describes the initial conditions satisfied by the return times of a return chain in a rooted tree with dynamics. When we construct a return chain, we can choose $l(0)$ (Proposition 2.13). We define a normal form for return chains by requiring that $l(0)=0$. This requirement normalizes the initial conditions of the return times.

Corollary 2.16. Let $(\mathrm{T}, \mathrm{F})$ be a rooted tree with dynamics such that $H \geq 1$. Let $\left(\mathrm{x}_{l(k)}\right)_{k \in \mathbb{Z}}$ be a return chain of $\mathbf{x}$ with return times $\left(n_{k}\right)$ generated by $r(k)$. If $l(0)=$ 0 , then $n_{k}=r(k)=1$ for all $k \leq 0$. Furthermore, if $\left(\mathrm{x}_{l(k)}\right)$ is minimal and $l(0)=0$, then $n_{1}=r(1)=1$.

Proof. Since $l(0)=0<H$, it follows from the proof of Proposition 2.15 that $n_{k}=1$ for all $k \leq 0$.

Now, assume that $\left(\mathrm{x}_{l(k)}\right)$ is minimal. We have $\mathrm{F}\left(\mathrm{x}_{H}\right)=\mathrm{x}_{0}=\mathrm{x}_{l(0)}$. Thus $l(1)=$ $H$, since $H$ is the least possible index of a vertex of $\mathbf{x}$ that could return to $\mathrm{x}_{0}$. Therefore, $n_{1}=r(1)=1$.

\section{VARIABLE- $r$ META-FibONACCI SEQUENCES}

In this section, we study variable- $r$ meta-Fibonacci sequences. In light of Lemma 2.9 and Corollary 2.16, we will only consider non-decreasing $r(k)$-bonacci sequences 
$\left(n_{k}\right)$ with $n_{k}=1$ for $k \leq 0$. We derive some estimates for $n_{k}$ based on bounds of $r(k)$ (\$3.2). We give two main estimates for $n_{k}$, a lower bound and an upper bound (Propositions 3.1 and 3.2 respectively). We also give some examples of variable- $r$ meta-Fibonacci sequences ( 3.1 ).

We say that an $r(k)$-bonacci sequence $\left(n_{k}\right)$ has a cascade of length $j \geq 1$ if $n_{k}=\cdots=n_{k+j-1}$ for some $k \geq 1$. Naturally, if $\left(n_{k}\right)$ is the sequence of return times of a return chain, then a cascade corresponds to a cascade of central returns.

Let $\lfloor\cdot\rfloor$ denote the greatest integer function.

Proposition 3.1. Let $\left(n_{k}\right)_{k \in \mathbb{Z}}$ be a non-decreasing $r(k)$-bonacci sequence such that $n_{k}=1$ for all $k \leq 0$. Let $J \in \mathbb{Z}^{+}$. If the length of every cascade of $\left(n_{k}\right)$ is bounded above by $J$, then

$$
n_{k} \geq 2^{\lfloor k /(J+2)\rfloor} \quad \text { for every } k \geq 1 .
$$

Proposition 3.2. Let $\left(n_{k}\right)_{k \in \mathbb{Z}}$ be a non-decreasing $r(k)$-bonacci sequence such that $n_{k}=1$ for all $k \leq 0$. Let $M \in \mathbb{Z}^{+}$. If $r(k+1) \leq M r(k)+1$ for every $k \geq 1$, then

$$
n_{k} \leq(M+1)^{k} \quad \text { for every } k \geq 1 \text {. }
$$

3.1. Examples. Before we prove the above estimates, we give some examples of $r(k)$-bonacci sequences. The most elementary example is when $r(1)=1$ and $r(k)=$ 2 for all $k \geq 2$. Then $n_{k}=u_{k+1}$, where $\left(u_{k}\right)$ is the usual Fibonacci sequence. Suppose that $r(k)=r \geq 2$ for all $k$ sufficiently large. Then up to re-indexing, the tail of $n_{k}$ is a generalized $r$-bonacci sequence for some initial conditions Mi.

If $r(k)=1$ for all $k$, then $n_{k}=1$ for all $k$. If $r(k)=1$ for all $k$ large, then $n_{k}$ is eventually constant. While this may seem like a trivial example, it corresponds to the return times of a periodic end (Lemma 2.10).

The following example shows that the return times of the Feigenbaum polynomial $[\mathrm{S}$ ] are $r(k)$-bonacci numbers.

Example 3.3. Let $r(k)=k$ for all $k \geq 1$. By an easy inductive argument, we find that $n_{k}=2^{k-1}$ for $k \geq 1$.

We can make $n_{k}$ grow linearly by taking $r(k)=1$ for many successive $k$.

Example 3.4. For $k \geq 2$, let $r(k)=2$ if $k=2^{m}$ for some $m \in \mathbb{Z}^{+}$, and let $r(k)=1$ otherwise.

\begin{tabular}{|c|c|c|c|c|c|c|c|c|c|c|c|c|}
\hline$k$ & 0 & 1 & 2 & 3 & 4 & 5 & 6 & 7 & 8 & 9 & $\cdots$ & 16 \\
\hline$r(k)$ & 1 & 1 & 2 & 1 & 2 & 1 & 1 & 1 & 2 & 1 & $\cdots$ & 2 \\
\hline$n_{k}$ & 1 & 1 & 2 & 2 & 4 & 4 & 4 & 4 & 8 & 8 & $\cdots$ & 16 \\
\hline
\end{tabular}

It is straightforward to show that $k / 2<n_{k} \leq k$ for $k \geq 1$.

The following is an example of an extended $r(k)$-bonacci sequence.

Example 3.5. Define $r(k)=2^{k-1}$ for $k \geq 1$.

\begin{tabular}{|c|c|c|c|c|c|c|c|c|c|c|c|}
\hline$k$ & $\leq 0$ & 1 & 2 & 3 & 4 & 5 & 6 & 7 & 8 & 9 & 10 \\
\hline$r(k)$ & 1 & 1 & 2 & 4 & 8 & 16 & 32 & 64 & 128 & 256 & 512 \\
\hline$n_{k}$ & 1 & 1 & 2 & 5 & 13 & 33 & 81 & 193 & 449 & 1025 & 2305 \\
\hline
\end{tabular}

It follows from Lemma 3.6 and Proposition 3.2 that $2<n_{k} / n_{k-1}<3$ for every $k \geq 3$. 
3.2. Estimates. We give some estimates on the terms of an $r(k)$-bonacci sequence based on bounds on $r(k)$. For a given $k$, the larger $r(k)$ is, the larger $n_{k}$ will be in comparison to $n_{k-1}$. However the exact relationship is subtle. We are particularly interested in closed-form bounds. That is, bounds for $n_{k}$ which are functions of $k$, but not of $n_{1}, \ldots, n_{k-1}$ or $r(k)$.

First, we recall the asymptotic growth rate of the $r$-bonacci numbers. Let $r \in \mathbb{Z}^{+}$. Define $\gamma_{r}$ as the unique root of

$$
z^{r}-z^{r-1}-\cdots-z-1
$$

such that $1 \leq\left|\gamma_{r}\right|<2$, where $|\cdot|$ denotes the complex norm. It is known that $\gamma_{r}$ is well defined and real for all $r$ [Mi, Eq. $\left.6^{\prime \prime}\right]$. For example, $\gamma_{2}=(1+\sqrt{5}) / 2$. Let $\left(u_{r, k}\right)_{k=1}^{\infty}$ be the $r$-bonacci numbers, then $\lim _{k \rightarrow \infty} u_{r, k+1} / u_{r, k}=\gamma_{r}$. The sequence $\left(\gamma_{r}\right)_{r=1}^{\infty}$ is strictly increasing, and $\lim _{r \rightarrow \infty} \gamma_{r}=2[\mathrm{Du}]$. We can compare the growth rate of an $r(k)$-bonacci sequence with bounded $r(k)$ to $\gamma_{r}$.

Let $\left(n_{k}\right)_{k \in \mathbb{Z}}$ be a non-decreasing $r(k)$-bonacci sequence such that $n_{k}=1$ for all $k \leq 0$. Let $R \in \mathbb{Z}^{+}$. If

$$
\liminf _{k \rightarrow \infty} r(k) \geq R, \quad \text { then } n_{k} \geq \text { const. } \gamma_{R}^{k} \quad \text { for all } k \geq 1
$$

and some positive constant [E3, Prop. 3.5]. A similar upper bound holds. If

$$
\limsup _{k \rightarrow \infty} r(k) \leq R, \quad \text { then } n_{k} \leq \text { const. } \gamma_{R}^{k} \text { for all } k \geq 1
$$

and some positive constant.

We now derive upper and lower bounds using weaker assumptions. The following lemma is the key observation about the growth of $n_{k}$ as a function of $r(k)$. We give a condition for $n_{k}$ to double (see Example 3.3).

Lemma 3.6 ([E3, Lem. 3.3]). Let $\left(n_{k}\right)_{k \in \mathbb{Z}}$ be an $r(k)$-bonacci sequence. If $r(k+1)=$ $r(k)+1$ for some $k$, then $n_{k+1}=2 n_{k}$.

Proof. By definition,

$$
n_{k+1}=\sum_{j=1}^{r(k+1)} n_{k+1-j}=n_{k}+\sum_{j=2}^{r(k)+1} n_{k+1-j}=n_{k}+\sum_{i=1}^{r(k)} n_{k-i}=2 n_{k} .
$$

There are $r(k)$-bonacci sequences where $\left(n_{k}\right)$ grows linearly (Example 3.4), or even slower [E3, Thm. 1]. This slow growth occurs when we have long cascades. Proposition 3.1 shows that this is the only way to obtain sub-exponential growth of $n_{k}$.

Proof of Proposition 3.1. First, consider the assumption that the length of cascades of $\left(n_{k}\right)$ is bounded above by $J$. It follows that for $k \geq 1$, the maximum number of consecutive $r(k)$ that equals 1 is also $J$. Hence, at least one of $r(k+1), \ldots$, $r(k+J+1)$ is larger than 1 for any $k \geq 0$.

Next fix $k \geq 0$. It suffices to show that $n_{k+J+2} \geq 2 n_{k}$. If $r(k+1)=1$ or $r(k+2)=1$, then $n_{k+J+2} \geq 2 n_{k}$ by the above observation about $r(k)$ and Lemma 3.6. By similar arguments, we can reduce to the case when $r(k+1) \geq r(k+2)>1$. This implies that $n_{k+1} \geq n_{k}+n_{k-1}$, and $n_{k+2} \geq n_{k+1}+n_{k}$. Therefore, $n_{k+J+2} \geq$ $n_{k+2} \geq 2 n_{k}+n_{k-1}>2 n_{k}$. 
The above estimate is sharp, as the following example shows. Fix $J \geq 1$. Define $r(k)=2$ if $k \equiv 0(\bmod J+2)$ and $k \geq 1$, and $r(k)=1$ otherwise. Then for $k \geq 1$, $n_{k}=2^{k /(J+2)}$ if $k \equiv 0(\bmod J+2)$ and $k \geq 1$, and $n_{k}=n_{k-1}$ otherwise.

Proposition 3.2 follows from the following lemma by induction. We derive an upper bound for the magnitude of $n_{k+1}$ relative to $n_{k}$.

Lemma 3.7. Let $\left(n_{k}\right)_{k \in \mathbb{Z}}$ be a non-decreasing $r(k)$-bonacci sequence with $n_{k}=1$ for $k \leq 0$. Let $M \in \mathbb{Z}^{+}$. If $r(k+1) \leq M r(k)+1$ for some $k$, then $n_{k+1} \leq(M+1) n_{k}$. Moreover, the equivalent statement with strict inequalities holds.

Proof. By definition,

$$
\begin{aligned}
n_{k+1} & =n_{k}+\sum_{j=2}^{r(k+1)} n_{k+1-j} \\
& \leq n_{k}+\sum_{i=1}^{M r(k)} n_{k-i} \quad \text { (by assumption) } \\
& =n_{k}+\sum_{m=0}^{M-1} \sum_{i=1}^{r(k)} n_{k-i-m r(k)} \\
& \leq n_{k}+\sum_{m=0}^{M-1} \sum_{i=1}^{r(k)} n_{k-i} \quad \text { (since the } n_{k} \text { are non-increasing) } \\
& =(1+M) n_{k} .
\end{aligned}
$$

Moreover, if $r(k+1)<M r(k)+1$, then the second line above is a strict inequality.

The above estimate is sharp. If $n_{k-M}=\cdots=n_{k}$ and $r(k+1)=M+1$ for some $k$, then $r(k+1)=M r(k)+1$ and $n_{k+1}=(M+1) n_{k}$.

The above upper bound is unexpectedly small. A constant function $r(k)$ generates a sequence $\left(n_{k}\right)$ which grows exponentially. A logarithmic $r(k)$ generates a sequence which grows linearly (Example 3.4). A priori, if $r(k)$ grows exponentially, then we might expect that $\left(n_{k}\right)$ would grow super-exponentially. However, Proposition 3.2 shows that it does not (see Example 3.5).

\section{Polynomial trees with dynamics}

In this section, we describe the construction of a tree with dynamics associated with a polynomial. We also prove our main theorem. Every polynomial of degree at least two is associated with a tree with dynamics. First, we define an abstract puzzle of a polynomial, which is a sequence of decompositions of the Julia set of the polynomial (\$.1). Every puzzle has a tree structure. A function that respects the puzzle structure gives rise to a tree with dynamics. We prove Theorem 1.2 . A polynomial of degree at least 2 has a standard puzzle. Green's function of a polynomial decomposes the plane into a puzzle, and the dynamics of the polynomial are compatible with the puzzle structure. We outline the construction of the Branner-Hubbard puzzle for a polynomial with a disconnected Julia set (\$4.2). This puzzle defines a canonical tree with dynamics of a polynomial with a disconnected Julia set. A polynomial with a connected Julia set has a tree with dynamics for each of its Yoccoz puzzles (\$4.3). We note some common properties of a standard 
polynomial tree with dynamics (4.4). Finally, we describe a generalized principal nest.

4.1. Puzzles. A puzzle is a sequence of Markov partitions in a general sense. We define an abstract puzzle of a polynomial. We have two main reasons for doing so. First, we wish to list common properties of polynomial puzzles. Second, we wish to isolate the properties we need to define a tree with dynamics.

Definition 4.1. Let $f$ be a polynomial of degree at least 2. A puzzle of $f$ is a sequence $\mathcal{P}=\left(\mathcal{P}_{l}\right)_{l \in \mathbb{Z}}$, where each $\mathcal{P}_{l}$ is an at most countable collection of disjoint non-empty subsets of $\mathbb{C}$ such that for each $l \in \mathbb{Z}$,

$$
\mathcal{K}(f) \subset \bigcup_{P \in \mathcal{P}_{l}} \bar{P}
$$

We call each $P \in \mathcal{P}_{l}$ a puzzle piece at depth $l$. We require that the puzzle pieces satisfy the following Markov properties:

(1) For any puzzle pieces $P_{1}, P_{2}$, there is a puzzle piece $P$ such that $P_{1} \cup P_{2} \subset P$.

(2) If $P \in \mathcal{P}_{l}$ for some $l$, then $P \subsetneq P^{\mathrm{p}}$ for some (unique) $P^{\mathrm{p}} \in \mathcal{P}_{l-1}$.

(3) If $P \in \mathcal{P}_{l}$ for some $l$, then $f(P)=Q$ and $f\left(P^{\mathrm{p}}\right)=Q^{\mathrm{p}}$, for some $Q \in \mathcal{P}_{L}$ with $L<l$.

There is a standard puzzle for each polynomial (of degree $\geq 2$ ), which is the Branner-Hubbard puzzle in the disconnected case ( $[\mathrm{BH}]$ and $[\mathrm{E} 2]$ ), or a Yoccoz puzzle in the connected case $([\underline{\mathrm{H}}$ and $[\mathrm{K}])$. However, the standard definition of a Yoccoz puzzle only includes $\mathcal{P}_{l}$ with $l \in \mathbb{N}$. So we modify the standard definition to define $\mathcal{P}_{l}$ for $l<0$ (Definition 4.13). These modifications produce a finite number of puzzle pieces with exceptional dynamics; they only satisfy a weakened version of condition 3:

$\left(3^{\prime}\right)$ If $P \in \mathcal{P}_{l}$ for some $l$, then $f(P) \subset Q \subset f\left(P^{\mathrm{p}}\right)$ for some $Q \in \mathcal{P}_{L}$ with $L<l$.

Definition 4.2. Let $\mathcal{P}=\left(\mathcal{P}_{l}\right)_{l \in \mathbb{Z}}$ be a puzzle a polynomial $f$. We define $\mathbf{T}(\mathcal{P})$, the genealogical tree of $\mathcal{P}$, by defining each puzzle piece as a vertex. We define the parent of $P$ as $P^{\mathrm{p}}$.

We define the dynamics on a tree of a puzzle. Notice that the above definition defines the ancestors of a puzzle piece.

Definition 4.3. Let $\mathcal{P}=\left(\mathcal{P}_{l}\right)_{l \in \mathbb{Z}}$ be a puzzle of a polynomial $f$. We define the induced dynamics $\mathrm{F}_{f}: \mathrm{T}(\mathcal{P}) \rightarrow \mathrm{T}(\mathcal{P})$ by $\mathrm{F}_{f}(P)=f(P)$ for non-exceptional puzzle pieces.

Assume that every puzzle piece with exceptional dynamics has an ancestor with non-exceptional dynamics. For a puzzle piece $P$ with exceptional dynamics, inductively assume that $\mathrm{F}_{f}\left(P^{\mathrm{p}}\right)$ is defined. Define $\mathrm{F}_{f}(P)=Q$, where $Q$ is the unique puzzle piece such that $f(P) \subset Q$ and $Q^{\mathrm{p}}=\mathrm{F}_{f}\left(P^{\mathrm{p}}\right)$.

We only used the Markov properties of the puzzle for the above definitions: puzzle pieces are ordered by inclusion, and $f$ respects this order.

Lemma 4.4. Let $\mathcal{P}$ be a puzzle of a polynomial $f$. If every puzzle piece with exceptional dynamics has an ancestor with non-exceptional dynamics, then $\left(\mathrm{T}(\mathcal{P}), \mathrm{F}_{f}\right)$ is a tree with dynamics. 
Proof. Definitions 4.2 and 4.3 certainly define a graph with a self-map. Markov property 1 implies that this graph is connected, and property 2 implies it has no non-trivial circuits. Hence, it is a tree.

Property 3 implies that $\mathrm{F}_{f}$ preserves children. We must show that $\mathrm{F}_{f}$ is well defined on puzzle pieces with exceptional dynamics. Suppose $P$ has exceptional dynamics. Inductively we may assume that we have defined $\mathrm{F}_{f}\left(P^{\mathrm{p}}\right)$, such that $f\left(P^{\mathrm{p}}\right) \subset \mathrm{F}_{f}\left(P^{\mathrm{p}}\right) \in \mathcal{P}_{L+1}$ for some $L$. It follows from Condition $\left(3^{\prime}\right)$ that $f(P) \subset$ $Q_{2} \subset f\left(P^{\mathrm{p}}\right)$ for some puzzle piece $Q_{2} \in \mathcal{P}_{l_{2}}$ with $l_{2} \leq L$. We can find $Q_{1} \in \mathcal{P}_{L}$ such that $f(P) \subset Q_{2} \subset Q_{1}$ and $Q_{1}^{\mathrm{p}}=\mathrm{F}_{f}\left(P^{\mathrm{p}}\right)$, by applying Condition (2) a finite number of times. If $Q_{1}^{\prime}$ is another such piece, then $f(P) \subset Q_{1} \cap Q_{1}^{\prime}$. So $Q_{1}=Q_{1}^{\prime}$ by disjointness of puzzle pieces at level $L$. Thus $Q_{1}$ is unique, and $\mathrm{F}_{f}(P)=Q_{1}$ is well defined.

Given a puzzle, we define a nest of puzzle pieces which corresponds to an end of the tree with dynamics.

Definition 4.5. Let $\mathcal{P}$ be a puzzle of a polynomial $f$. A nest of puzzle pieces of $f$ is a sequence $\left(P_{l}\right)_{l \in \mathbb{N}}$ such that each $P_{l} \in \mathcal{P}_{l}$ and $P_{l}=P_{l+1}^{\mathrm{p}}$. An extended nest is the analogous sequence with $l \in \mathbb{Z}$.

Let $\left(P_{l}\right)$ be an extended nest of puzzle pieces of some puzzle $\mathcal{P}$ of a polynomial $f$. Let $\left(\mathrm{x}_{l}\right)$ be the extended end in the tree with dynamics $(\mathrm{T}, \mathrm{F})=\left(\mathrm{T}(\mathcal{P}), \mathrm{F}_{f}\right)$ that corresponds to $\left(P_{l}\right)$. Note that it is possible that $\mathrm{F}^{n}\left(\mathrm{x}_{l}\right)=\mathrm{x}_{m}$, but $f^{n}\left(P_{l}\right) \subsetneq P_{m}$ for some indices. For $l \in \mathbb{Z}$ and $n \geq 1$, we say $f^{n}\left(P_{l}\right)$ returns if $\mathrm{F}^{n}\left(\mathrm{x}_{l}\right)$ returns. We define the first return time of $P_{l}$ as the first return time of $\mathrm{x}_{l}$.

Definition 4.6. Let $\left(P_{l}\right)_{l \in \mathbb{Z}}$ be an extended nest of puzzle pieces of a polynomial $f$. Let $\left(\mathrm{T}(\mathcal{P}), \mathrm{F}_{f}\right)$ be the tree with dynamics of $\mathcal{P}$. A return nest is a sub-nest $\left(P_{l(k)}\right)_{k \in \mathbb{Z}}$ such that

$$
\mathrm{F}_{f}^{n_{k}}\left(P_{l(k)}\right)=P_{l(k-1)}
$$

where $n_{k}$ is the first return time of $P_{l(k)}$. We call $\left(n_{k}\right)_{k \in \mathbb{Z}}$ the return times of the return nest. If $l(k)=\min \left\{l: P_{l(k-1)}\right.$ is the first return of $\left.P_{l}\right\}$ for all $k>0$, then we call the return nest minimal.

If $f^{n}\left(P_{l(k)}\right)$ has exceptional dynamics for some $k$ and some $n=0, \ldots, n_{k}-1$, then we only have that $f^{n_{k}}\left(P_{l(k)}\right) \subset P_{l(k-1)}$. Otherwise, we have $f^{n_{k}}\left(P_{l(k)}\right)=P_{l(k-1)}$. A nest of standard puzzle of $f$ has at most one exceptional puzzle piece. In a Yoccoz puzzle, if $l(k-1)=0$, then we may have $f^{n_{k}}\left(P_{l(k)}\right) \subsetneq P_{l(k-1)}$. A principal nest (Definition 4.19) is a special case of a return nest.

Lemma 4.7. Let $f$ be a polynomial of degree $\geq 2$. Let $\mathcal{P}$ be a puzzle of $f$ and $(\mathrm{T}, \mathrm{F})=\left(\mathrm{T}(\mathcal{P}), \mathrm{F}_{f}\right)$. The following chart is a dictionary between the dynamical systems $(\mathcal{P}, f)$ and $(\mathrm{T}, \mathrm{F})$ :

\begin{tabular}{|l|l|}
\hline$(\mathcal{P}, f)$ & $(\mathrm{T}, \mathrm{F})$ \\
\hline$P$ a puzzle piece & $\mathrm{v}$ a vertex \\
\hline$\left(P_{l}\right)_{l \in \mathbb{Z}}$ an extended nest of puzzle pieces & $\left(\mathrm{x}_{l}\right)_{l \in \mathbb{Z}}$ an extended end \\
\hline$\left(P_{l(k)}\right)_{k \in \mathbb{Z}}$ a return nest & $\left(\mathrm{x}_{l(k)}\right)_{k \in \mathbb{Z}}$ a return chain \\
with return times $\left(n_{k}\right)$ & with return times $\left(n_{k}\right)$ \\
\hline
\end{tabular}


Proof. The only non-obvious statement is that the return times of a return nest and the corresponding return chain are equal. Observe that the definition of return nest (Definition 4.6) uses the tree with dynamics. Therefore, the return times are the same.

We prove our main theorem: The return times of any return nest are variable- $r$ meta-Fibonacci numbers.

Proof of Theorem 1.2. Theorem 2.11 applies to any tree with dynamics of a polynomial by Proposition 4.17. By the dictionary (Lemma4.7), a return nest corresponds to a return chain and the return times are the same.

When we construct a standard puzzle of a polynomial $\mathcal{P}$, then $\mathcal{P}_{l}$ always contains exactly one puzzle piece for $l \leq 0$ (Proposition 4.17). Hence we can say more about the return times of a nest from a standard puzzle.

Corollary 4.8. Let $\mathcal{P}$ be a puzzle of $f$ such that $\mathcal{P}_{l}$ contains exactly one puzzle piece for $l \leq 0$. If $\left(n_{k}\right)$ are the return times of a return nest $\left(P_{l(k)}\right)$, then $n_{k}=r(k)=1$ for all $k \leq K$ for some $K \in \mathbb{Z}$. If $l(0)=0$, then $K=0$. Furthermore, if $l(0)=0$ and the nest is minimal, then $K=1$.

Proof. Say $\mathcal{P}_{0}=\left\{P_{0}\right\}$. Since $\mathcal{P}_{l}$ contains exactly one puzzle piece for $l \leq 0, P_{0}$ is the root of $\left(\mathbf{T}(\mathcal{P}), \mathrm{F}_{f}\right)$. Apply Proposition 2.15. For the last parts, use Corollary 2.16 .

Remark 4.9. Theorem 1.2 and Corollary 4.8 give necessary conditions for the return times of a return nest. It is natural to ask what are sufficient conditions? Moreover, are these conditions different for polynomials with a connected Julia set? with a disconnected Julia set? or for real polynomials? However, we do not address these questions at this time, since it would require a more in depth and technical analysis of a tree with dynamics than we wish to present in this paper.

4.2. Polynomials with disconnected Julia sets. We now turn to describing the standard puzzle of a polynomial. First we recall some facts that we will use in both the connected and disconnected cases.

Let $f$ be a polynomial of degree $d \geq 2$. Let $g$ denote Green's Function of $f$. We use $g$ to decompose the plane. Recall that $g(z) \geq 0$ for all $z \in \mathbb{C}$ and $g(z)=0$ if and only if $z \in \mathcal{K}(f)$. The functional equation $g(f)=d \cdot g$ is satisfied by $f$ and $g$. The critical points of $g$ are the critical points of $f$ and the iterated pre-images of critical points of $f$. An equipotential is a level set of $g$ of the form $\{z \in \mathbb{C}: g(z)=\lambda>0\}$. By the functional equation, $f$ maps equipotentials to equipotentials. Equipotentials have a dynamical definition (see $[\mathrm{B}]$ ). It follows that the tree with dynamics of a polynomial with a disconnected Julia set is a topological invariant.

Let $\varphi$ be some Böttcher function of $f$. If the Julia set of $f$ is connected, then $\varphi^{-1}: \mathbb{C} \backslash \overline{\mathbb{D}} \rightarrow \mathbb{C} \backslash \mathcal{K}(f)$ is a conformal isomorphism. An external ray is a set of the form $\varphi^{-1}\left\{s e^{2 \pi i \theta}: 0<s<\infty\right\}$ for some $\theta \in \mathbb{R} / \mathbb{Z}$. An external ray is rational if $\theta \in \mathbb{Q} / \mathbb{Z}$.

We outline the dynamical decomposition of the plane using $g$ for a polynomial with a disconnected Julia set. Fix a polynomial $f$ of degree $d \geq 2$ with a disconnected Julia set. We distinguish all equipotentials whose grand orbit contains a critical point of $f$. There are countably many such equipotentials, say $\left\{E_{l}\right\}_{l \in \mathbb{Z}}$. Index them so that $g\left|E_{l}<g\right| E_{l-1}, E_{l}$ is a Jordan curve for $l \leq 0$, and $E_{1}$ is not a 
Jordan curve (so it contains a subset homeomorphic to a figure-8). Let $H$ be the number of orbits of $\left\{E_{l}\right\}_{l \in \mathbb{Z}}$ under $f$. If $f$ has $e$ distinct critical points that escape to infinity, then $H \leq e$. It is possible that $H<e$ if $f$ has two escaping critical points $c$ and $c^{\prime}$ such that $g(c)=d^{n} g\left(c^{\prime}\right)$ for some $n \in \mathbb{Z}$. From the functional equation and the indexing of $E_{l}$, it follows that $f\left(E_{l}\right)=E_{l-H}$ for all $l$. In fact, $H$ is equivalent to the $H$ that is used in trees with dynamics. Notice that $H$ is positive.

Define $U_{l}=\left\{z: g(z)<g \mid E_{l}\right\}$. For $l \leq 0, U_{l}$ is a single topological disk (defined as a simply connected open set). For all $l, U_{l}$ is the disjoint union of finitely many topological disks $\left\{P_{l}^{i}\right\}$. We define each of these disks as a puzzle piece of $f$ at depth $l$, and $\mathcal{P}_{l}=\left\{P_{l}^{i}\right\}$. The Branner-Hubbard puzzle is $\mathcal{P}=\left(\mathcal{P}_{l}\right)[\mathrm{BH}$, Ch. 3]. It is known that the Markov properties hold for these puzzle pieces.

Definition 4.10. Let $f$ be a polynomial of degree at least 2 with a disconnected Julia set. Let $\mathcal{P}$ be the Branner-Hubbard puzzle of $f$. We define the canonical tree with dynamics of $f$ as $\left(\mathrm{T}(\mathcal{P}), \mathrm{F}_{f}\right)$.
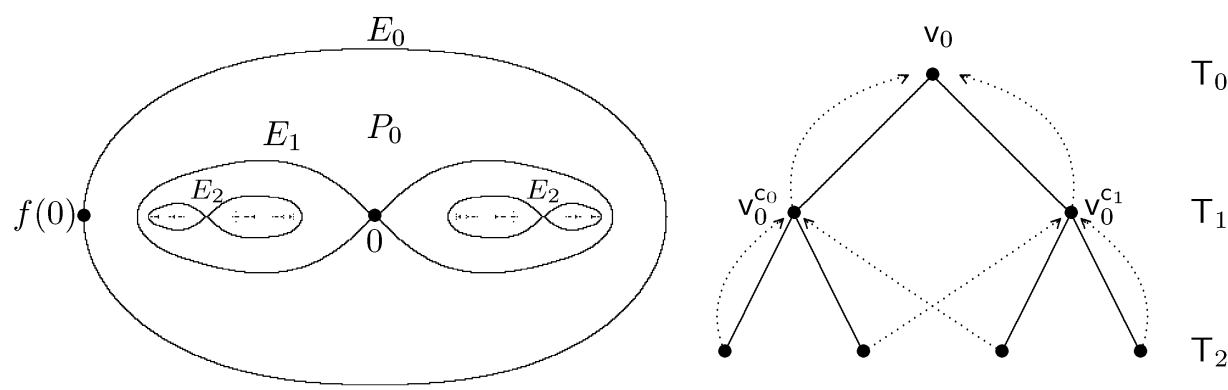

FIGURE 4. Equipotentials of a quadratic polynomial with a disconnected Julia set and its tree with dynamics.

Example 4.11. Let $f(z)=z^{2}+c$ have a disconnected Julia set. There is exactly one escaping critical point of $f$, namely 0 . So $H=1$. The equipotential that contains 0 is $E_{1}$. Each equipotential $E_{-l}=f^{l+1}\left(E_{1}\right)=\left\{z: g(z)=2^{l+1} g(0)\right\}$ is an analytic Jordan curve for $l \in \mathbb{N}$. Hence, $T_{-l}$ is a single vertex for $l \in \mathbb{N}$. Since 0 is a simple critical point, $f$ is locally two-to-one near 0 . Thus, $E_{1}$ is homeomorphic to a figure-8: two Jordan curves pasted at 0 . There are exactly two components of $E_{2}=f^{-1}\left(E_{1}\right)=\left\{z: g(z)=2^{-1} g(0)\right\}$, one inside each loop of $E_{1}$. Thus, there are exactly two puzzle pieces of $f$ at depth 1 . So in the tree with dynamics of $f$, $\mathrm{T}_{0}=\left\{\mathrm{v}_{0}\right\}$ and $\mathrm{T}_{1}=\left\{\mathrm{v}_{0}^{\mathrm{c}_{0}}, \mathrm{v}_{0}^{\mathrm{c}_{1}}\right\}$. For $l>1, f$ maps $E_{l}$ onto $E_{l-1}$. This map is one-to-one, since there are no critical points in the bounded components of $\mathbb{C} \backslash E_{l}$. Therefore each component of $E_{l}$ is homeomorphic to a figure-8. It follows that there are exactly two components of $E_{l+1}$ nested inside each component of $E_{l}$; one in each loop of the figure-8. Thus if $\vee \in \mathrm{T}_{l}$ for some $l \in \mathbb{N}$, then $\vee$ has exactly two children, which are mapped by $F$ onto the children of $F(v)$. It follows that $(T, F)$ is the tree with dynamics from Example 2.5.

A child of a puzzle piece $P \in \mathcal{P}_{l}$ is a $P^{\mathrm{c}} \in \mathcal{P}_{l+1}$ such that $P^{\mathrm{c}} \subset P$. Define $A=P \backslash \bigcup \overline{P^{c}}$ as an annulus of $f$. So, there is a one-to-one correspondence between 
annuli and puzzle pieces. Since $f$ preserves children, it follows that $f$ maps each annulus onto an annulus. Let $A_{i}=P_{i} \backslash \bigcup \overline{P_{i}^{c}}$ for $i=1,2$. Then $f\left(A_{1}\right)=f\left(A_{2}\right)$ if and only if $f\left(P_{1}\right)=f\left(P_{2}\right)$. Therefore, we could have just as well used annuli instead of puzzle pieces to define the puzzle.

Remark 4.12. Because of the correspondence between puzzle pieces and annuli, we could have defined the vertices of the tree as annuli. Thus, Definition 4.10 gives the same tree with dynamics as [E2, Def. 3.7].

4.3. Polynomials with connected Julia sets. When $f$ is a polynomial with a connected Julia set, we choose a Yoccoz puzzle $([\mathrm{H}]$ and $[\mathrm{K}]$ ). We outline the construction here. All the equipotentials of $f$ are Jordan curves, so we use external rays to separate the Julia set.

Fix a polynomial $f$ of degree $d \geq 2$ with a connected Julia set. Choose a finite set $\alpha_{1}, \ldots, \alpha_{m} \in \mathcal{J}(f)$ such that at least one rational ray lands on each $\alpha_{i}$, at least two distinct rational rays land on some $\alpha_{j}$, and $f\left(\left\{\alpha_{i}\right\}\right) \subset\left\{\alpha_{i}\right\}$. For quadratic polynomials, one usually chooses $\left\{\alpha_{i}\right\}=\{\alpha\}$, where $\alpha$ is a repelling fixed point that is the landing point of at least two distinct external rays. In general, we could choose a finite number of repelling periodic cycles. Choose $\lambda>0$. The equipotentials $\left\{z: g(z)=d^{l} \lambda\right\}$ and the pre-images of the external rays that land on $\left\{\alpha_{i}\right\}$ partition the plane. We define the Yoccoz puzzle of $f$ determined by $\left\{\alpha_{i}\right\}$ and $\lambda$. Define $U_{1}=\{z: g(z)<\lambda\}$, and $\Gamma_{1}$ as the external rays that land on $\alpha_{1}, \ldots, \alpha_{m}$ including the landing points. We define a sequence of open sets $\left(U_{l}\right)_{l=1}^{\infty}$ and graphs $\left(\Gamma_{l}\right)_{l=1}^{\infty}$, by $U_{l+1}=f^{-1}\left(U_{l}\right)$ and $\Gamma_{l+1}=f^{-1}\left(\Gamma_{l}\right)$. For technical reasons, define $U_{-l}=\left\{z: g(z)<d^{l} \lambda\right\}$ and $\Gamma_{-l}=\emptyset$ for $l \in \mathbb{N}$. A connected component $P_{l}^{i}$ of $U_{l} \backslash \Gamma_{l}$ is a puzzle piece of $f$ at depth $l$, and $\mathcal{P}_{l}=\left\{P_{l}^{i}\right\}$. Define the Yoccoz puzzle by $\mathcal{P}=\left(\mathcal{P}_{l}\right)$. Note that what we call depth $l$, previous authors call depth $l-1$. The Markov properties hold for pieces at any level $l \geq 1$ [K, $\$ 12]$.

Puzzle pieces at depth $l \leq 0$ have not been defined in the existing literature. Each of these puzzle pieces is bounded by an equipotential, but not by any external rays. Hence, there is only one puzzle piece at any depth $l \leq 0$, which is a topological disk. It is easy to check the Markov properties hold for these pieces. A puzzle piece at depth 1 may have exceptional dynamics. Let $\mathcal{P}_{0}=\left\{P_{0}\right\}$. If $P$ is a puzzle piece at depth 1 , then $f(P) \subset P_{0}$. However in general, $f(P) \neq P_{0}$. Even if $f(P)=P_{0}$, the map $f: P \rightarrow P_{0}$ is not necessarily proper. From Lemma 4.4, we have $F_{f}(P)=P_{0}$. The exceptional nature of these pieces does not affect return times.

Definition 4.13. Let $f$ be a polynomial with a connected Julia set. Let $\mathcal{P}$ be some Yoccoz puzzle of $f$. We define the standard tree with dynamics with respect to $\mathcal{P}$ as $\left(\mathrm{T}(\mathcal{P}), \mathrm{F}_{f}\right)$.

Remark 4.14. The tree with dynamics of a polynomial with a connected Julia set depends on the choice of $\mathcal{P}$. Specifically on the $\left\{\alpha_{i}\right\}$ that are chosen in the construction $\mathcal{P}$. Hence the tree with dynamics of a polynomial with a connected Julia set is not canonical. However it does not depend on the choice of $\lambda$.

Example 4.15. Let $f(z)=z^{2}+c$ have a connected Julia set. Assume exactly 2 external rays land on some fixed point $\alpha$. We outline the construction of the first few levels of a Yoccoz puzzle of $f$ and the associated tree with dynamics, see Figure 5. We choose $\left\{\alpha_{i}\right\}=\{\alpha\}$, and any $\lambda>0$. By definition, $U_{-l}=\left\{z: g(z)<2^{l+1} \lambda\right\}$ and $\Gamma_{-l}=\emptyset$ for $l \in \mathbb{N}$. Thus $\mathcal{P}_{-l}$ is a single puzzle piece $P_{-l}$, and $\mathrm{T}_{-l}=\left\{\mathrm{v}_{-l}\right\}$ 

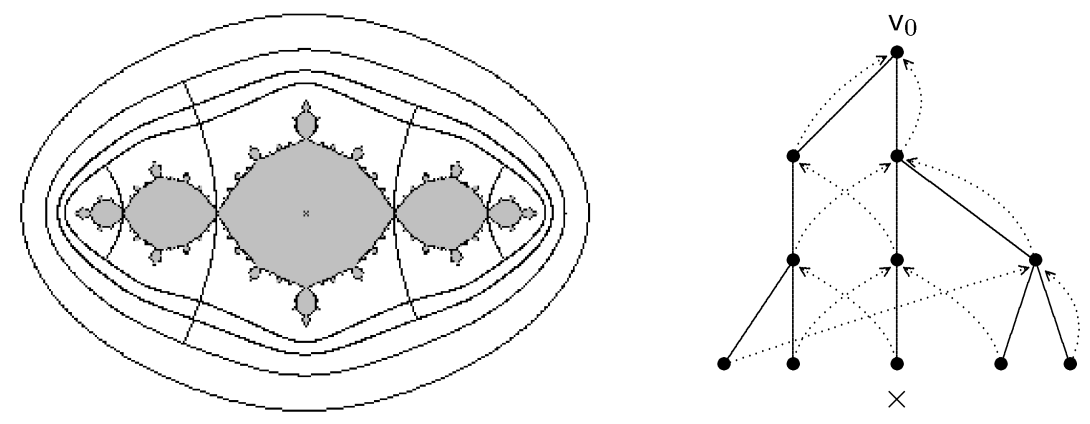

Figure 5. A Yoccoz puzzle of the Julia set of $z^{2}-1$ and its tree with dynamics. The end which corresponds to the nest of the critical point $(0)$ is indicated by the symbol $\times$.

for $l \in \mathbb{N}$. We have $\Gamma_{1}=\left\{\frac{1}{3}, \frac{2}{3}\right\}$, and these two external rays separate $U_{1}$ into two puzzle pieces, say $P_{1}^{0}$ which contains 0 , and $P_{1}^{1}$. We have $f\left(P_{1}^{1}\right) \subsetneq P_{0}$ and $f\left(P_{1}^{0}\right)=P_{0}$. However, the map $f: P_{1}^{0} \rightarrow P_{0}$ is not proper. Next, $\Gamma_{2}=\Gamma_{1} \cup\left\{\frac{1}{6}, \frac{5}{6}\right\}$. So $P_{1}^{0}$ has two children, say $P_{2}^{0} \ni 0$ and $P_{2}^{1}$, and $P_{1}^{1}$ has only one child, $P_{2}^{2}$. So $f\left(P_{2}^{0}\right)=P_{1}^{1}, f\left(P_{2}^{1}\right)=P_{1}^{0}, f\left(P_{2}^{2}\right)=P_{1}^{0}$. Finally, $\Gamma_{3}=\Gamma_{2} \cup\left\{\frac{1}{12}, \frac{11}{12}, \frac{5}{12}, \frac{7}{12}\right\}$. This gives $P_{2}^{1}$ and $P_{2}^{2}$ two children each, and $P_{2}^{0}$ one child.

In the connected case, an annulus of $f$ at depth $l$ is the difference of nested puzzle pieces $P^{\mathrm{p}} \backslash \bar{P}$ where $P$ is a puzzle piece at depth $l$. A complication can arise. An annulus $A=P^{\mathrm{p}} \backslash \bar{P}$ is called degenerate if $\partial P \cap \partial P^{\mathrm{p}} \neq \emptyset$. A degenerate annulus is not doubly connected, but is the union of topological disks. This complication is a serious concern in modulus estimates (see $[\mathrm{H}, \S 9]$ ), but does not affect return times. Annuli are mapped onto annuli by $f$, except possibly for those at depth 1 . Let $A_{i}=P_{i}^{\mathrm{p}} \backslash \overline{P_{i}}$ for $i=1,2$. If $f\left(A_{1}\right)=A_{2}$, then $f\left(P_{1}^{\mathrm{p}}\right)=P_{2}^{\mathrm{p}}$ by the disjointness property of puzzle pieces.

Remark 4.16. As in the disconnected case, if we use annuli instead of puzzle pieces, we obtain the same tree with dynamics of $f$.

4.4. Properties of polynomial trees with dynamics. We note some properties satisfied by any tree with dynamics of a standard puzzle of a polynomial. The following properties follow immediately from the construction of a standard tree with dynamics of a polynomial (Definitions 4.10 and 4.13). Compare to [E2, Def. 3.2].

Proposition 4.17. If $(\mathrm{T}, \mathrm{F})$ is a tree with dynamics of a polynomial with respect to a standard puzzle, then the following hold.

(1) Every vertex of $\mathrm{T}$ has at least one child. That is, T has no leaves.

(2) Every vertex of $\mathrm{T}$ has only finitely many children. That is, $\mathrm{T}$ is locally finite.

(3) There is a root of $\mathrm{T}$.

(4) The integer $H$ such that $\mathrm{F}\left(\mathrm{T}_{l}\right)=\mathrm{T}_{l-H}$ is positive. Moreover $H=1$ for any tree with dynamics of a polynomial with a connected Julia set.

In this paper, the construction of a tree with dynamics from a puzzle is purely set theoretic. We only use the Markov properties of the puzzle. We never used the 
topological properties of puzzle pieces of a polynomial. Thus, the above proposition is a list of the properties of a tree with dynamics of a polynomial, which follow from the set-theoretic properties of a standard puzzle. These standard puzzles have some useful additional properties; all of their puzzle pieces are open, simply connected, and pre-compact. Also, a polynomial restricted to one of its puzzle pieces is a proper map, with at most finitely many exceptions. Notice that we never use these additional properties in this paper.

Axioms for the tree with dynamics of a polynomial with a disconnected Julia set are known. These axioms are complete in the sense that they are necessary and sufficient conditions for a tree with dynamics to be the tree with dynamics of a polynomial with a disconnected Julia set. These axioms were first given by the author in [E2, Def. 4.7], where their necessity was demonstrated. They were also shown to be sufficient for certain trees with dynamics [E2, Thm. 9.4]. L. DeMarco and C. McMullen showed that these axioms are sufficient (using different notation) DeMc. To define these complete axioms we need to keep track of some topological information. Specifically, the local degree of the polynomial restricted to each of its puzzle pieces. A formula for the number of children of each puzzle piece can then be deduced from the Riemann-Hurwitz formula for domains [E2, Def. 4.6].

Remark 4.18. To the best of the author's knowledge, complete axioms for a tree with dynamics of a polynomial with a connected Julia set are not known. There are two obvious differences from the disconnected case. The number of children of the root $\mathrm{v}_{0}$ can be arbitrarily high (depending on the choice of $\alpha_{1}, \ldots, \alpha_{m}$ ). The axioms for vertices at level 1 are not clear, since the polynomial restricted to a puzzle piece at level 1 is not necessarily proper. For all other levels, the polynomial restricted to each puzzle piece is proper. Thus, the conditions of [E2, Def. 4.6] must hold.

We now describe how the dynamics of a nest corresponds to the dynamics on a Julia set. Let $f$ be a polynomial and $\mathcal{P}$ a puzzle of $f$. For $z_{0} \in \mathcal{K}(f)$, a nest of $z_{0}$ is a nest of puzzle pieces $\left(P_{l}\right)$ such that $z_{0} \in \overline{P_{l}}$ for every $l$. If we regard a return of $z_{0}$ to $P_{l+1}$ as "closer" than a return to $P_{l}$, then the return times of a return nest can be thought of as generalized closest return times.

Let $\left(P_{l}\right)$ be a nest of puzzle pieces from the Branner-Hubbard puzzle of $f$ (Definition 4.10). Then $\bigcap_{l \in \mathbb{N}} \bar{P}_{l}$ is a connected component of $\mathcal{K}(f)$. So each point $z_{0} \in \mathcal{K}(f)$ lies in a unique nest. Thus the dynamics of $z_{0}$ are coded by the dynamics of a unique end of the tree with dynamics. Moreover, if $\mathcal{J}(f)$ is a Cantor set, then there is a one-to-one correspondence between points of $\mathcal{J}(f)$ and ends of the tree with dynamics.

Let $\mathcal{P}$ be a Yoccoz puzzle of $f$ (Definition 4.13). Let $z_{0} \in \mathcal{K}(f)$. If $z_{0}$ is not a pre-image of one of the $\alpha_{i}$ used to define the Yoccoz puzzle, then $z_{0}$ lies in a unique nest $\left(P_{l}\right)$. Although there is no obvious geometric interpretation of $\bigcap_{l \in \mathbb{N}} \overline{P_{l}}$. If $z_{0}$ is the pre-image of one of the $\alpha_{i}$ used to define the Yoccoz puzzle, then $z_{0} \in \bigcap_{l \in \mathbb{N}} \overline{P_{l}}$ for several nests $\left(P_{l}\right)$. Thus the dynamics of every point of $\mathcal{K}(f)$ are coded by an end of the tree with dynamics. For all but countably many of the points of $\mathcal{K}(f)$, this coding is unique.

A nest of puzzle pieces that contains a critical point is of special interest. 
Definition 4.19. Let $\left(P_{l}\right)$ be an extended nest of puzzle pieces of a polynomial $f$. Suppose that there is a critical point of $f$ in every $P_{l}$. A principal nest is a minimal return nest of $\left(P_{l}\right)$.

In terms of Markov properties, there is no difference between principal nests and return nests. The return times of both are $r(k)$-bonacci numbers. However, topologically they are different. A principal nest can be to used estimate the moduli of the annuli of its nest $\mathrm{BH}, \mathrm{Ch} .4 .5]$. A general return nest cannot be used in this way.

The concept of a principal nest appeared in the literature before the terminology was set. Branner and Hubbard introduced tableaux [BH, Ch. 4.2]. For a polynomial with exactly one persistent critical point, a tableau keeps track of the return times of a principal nest of the persistent critical point with $l(0)=0$. Later the zigzag pattern that defined the returns was called the critical staircase of the tableau $[\mathrm{B}$. Lyubich suggested a choice for $l(0)$, and we have generalized the terminology he introduced in [L, §3]. A standard puzzle of polynomial with a unique persistent critical point has a principal nest, which depends only on the choice of $l(0)$ (Proposition 2.13).

A principal nest can sometimes be used to find domains where a polynomial is renormalizable in the sense of Douady and Hubbard $[\mathrm{DH}]$. Let $\left(P_{l(k)}\right)_{k \in \mathbb{Z}}$ be a principal nest of a polynomial $f$. Then $f \mid P_{l(k)}$ is not univalent for any $k$. For a Branner-Hubbard puzzle, it is clear that $\left(f^{n_{k}} ; P_{l(k)}, P_{l(k-1)}\right)$ is polynomial-like (of some degree $\geq 2$ ) for every $k \in \mathbb{Z}$. A polynomial of the form $f(z)=z^{d}+c$ $(d \geq 2, c \in \mathbb{C})$ is called unicritical. Lyubich showed that for a Yoccoz puzzle of an unicritical polynomial, there is a choice of $l(0)$ for the principal nest such that $\left(f^{n_{k}} ; P_{l(k)}, P_{l(k-1)}\right)$ is polynomial-like for every $k \geq 1[\mathrm{~L}$, Prop. 3.1].

\section{ACKNOWLEDGEMENTS}

Portions of this paper are based upon my Ph. D. thesis. I am indebted to Ricardo Pérez-Marco for his guidance.

The computer generated images of Julia sets were created using Mandel by Wolf Jung (http://www.mndynamics.com), and DH-Drawer by Arnaud Cheritat (http://www.math.univ-toulouse.fr/ ${ }^{\sim}$ cheritat/).

\section{REFERENCES}

[B] Bodil Branner, Puzzles and para-puzzles of quadratic and cubic polynomials, Complex Dynamical Systems (Robert L. Devaney, ed.), Proc. Sympos. Appl. Math., vol. 49, AMS, 1994, pp. 31-67. MR1315533

[BH] Bodil Branner and John H. Hubbard, Iteration of cubic polynomials, part II: patterns and parapatterns, Acta Math. 169 (1992), 229-325. MR1194004 (94d:30044)

[CCT] Joseph Callaghan, John J. Chew, III, and Stephen M. Tanny, On the behavior of a family of meta-Fibonacci sequences, SIAM J. Discrete Math. 18 (2004), no. 4, 794-824. MR2157827(2006c:11012)

[CG] Lennart Carleson and Theodore W. Gamelin, Complex Dynamics, Springer-Verlag, 1993. MR.1230383 (94h:30033)

[DeMc] Laura G. DeMarco and Curtis T. McMullen, Trees and the dynamics of polynomials, to appear in Ann. Sci. École Norm. Sup.

[DH] Adrien Douady and John Hamal Hubbard, On the dynamics of polynomial-like mappings, Ann. Sci. École Norm. Sup. (4) 18 (1985), no. 2, 287-343. MR816367 (87f:58083)

[Du] François Dubeau, On r-generalized Fibonacci numbers, Fibonacci Quart. 27 (1989), no. 3, 221-229. MR:1002065 (90g:11022) 
[E1] Nathaniel D. Emerson, Dynamics of polynomials whose Julia set is an area zero Cantor set, $\mathrm{Ph}$. D. thesis, 2001.

[E2] Dynamics of polynomials with disconnected Julia sets, Discrete Contin. Dyn. Syst. 9 (2003), no. 4, 801-834. MR.1975358 (2004m:37083)

[E3] , A family of meta-Fibonacci sequences defined by variable-order recursions, J. Integer Seq. 9 (2006), no. 1, Article 06.1.8, 21 pp. (electronic). MR2211161

$[\mathrm{H}] \quad$ J. H. Hubbard, Local connectivity of Julia sets and bifurcation loci: three theorems of J.-C. Yoccoz, Topological Methods in Modern Mathematics (Stony Brook, NY, 1991), Publish or Perish, Houston, TX, 1993, pp. 467-511. MR.1215974 (94c:58172)

[K] Jan Kiwi, Rational rays and critical portraits of complex polynomials, Ph. D. thesis, 1997. Stony Brook IMS pre-print 97-15.

[L] Mikhail Lyubich, Dynamics of quadratic polynomials. I, II, Acta Math. 178 (1997), no. 2, 185-247, 247-297. MR1459261 (98e:58145)

[LM] Mikhail Lyubich and John Milnor, The Fibonacci unimodal map, J. Amer. Math. Soc. 6 (1993), no. 2, 425-457. MR 1182670 (93h:58080)

[Mi] E. P. Miles, Jr., Generalized Fibonacci numbers and associated matrices, Amer. Math. Monthly 67 (1960), no. 8, 745-752. MR0123521(23 A846)

[P-M] Ricardo Pérez-Marco, Degenerate conformal structures, manuscript, 1999.

[S] Dennis Sullivan, Bounds, quadratic differentials, and renormalization conjectures, American Mathematical Society centennial publications, Vol. II (Providence, RI, 1988), AMS, Providence, RI, 1992, pp. 417-466. MR1184622 (93k:58194)

Department of Mathematics, University of Southern California, Los Angeles, CalIFORNIA 90089

E-mail address: nemerson@usc.edu 